\title{
O uso de aplicativos de saúde para dispositivos móveis como fontes de informação e educação em saúde
}

The use of health applications for mobile devices as sources of

information and education in healthcare

Ana Rachel Fonseca de Oliveira ${ }^{1}$, Maria Simone de Menezes Alencar ${ }^{2}$

${ }^{1}$ Universidade do Estado do Rio de Janeiro

${ }^{2}$ Universidade Federal do Estado do Rio de Janeiro

\section{RESUMO}

Os aplicativos para dispositivos móveis são novíssimas fontes de informação utilizadas fortemente por indivíduos hiperconectados, a chamada "geração polegar", que além do uso recreativo recorre a estas tecnologias móveis em um contexto educacional inovando o esquema de ensino-aprendizagem. O artigo propõe que a biblioteca universitária seja utilizada como um espaço de ensino e incentivo ao uso de dispositivos móveis como ferramentas educativas, visto que o uso em massa de tablets e especialmente de smartphones reforçam o conceito de aprendizagem móvel e ampliam suas possíveis funções dentro do espaço físico da biblioteca. $\mathrm{Na}$ realidade de uma biblioteca universitária que tem como público-alvo alunos da área de saúde e, principalmente de Medicina, focaremos nos aplicativos de saúde como fontes de informação, comunicação e educação. Dentro deste cenário de inovações tecnológicas que modificam as formas de acesso e uso de informação científica, o bibliotecário deve assumir o papel de mediador e facilitador entre estes avanços tecnológicos e seus usuários em potencial.

PALAVRAS-ChAVE: Aplicativos móveis. Aprendizagem móvel. Competência informacional. Dispositivos móveis.

\section{ABSTRACT}

Applications for mobile devicesarebrand new sources of information heavily used by hyperconnectedindividuals, the socalled "thumb generation", who, going beyond recreational use, refer to these mobile technologies in an educational context, innovating the teaching-learning process. This paper posits that university libraries should be used as spaces for teaching and encouraging the use of mobile devices as educational tools, since the pervasiveness of tablets and especially smartphones strengthen the concept of mobile learning and widen their possible functions inside the physical space of the library. Taking into consideration the reality of a university library whose patronsarehealth and medicinemajors, our focus will be on health applications used as sources of information, communication and education. In this scenario of technological innovations that alter the ways to access and use scientific information, the librarian should take on the role of the mediator and facilitator between these technological advances and their potential users.

KEYWORDS: Mobile applications. Mobile learning. Information literacy. Mobile devices.

\section{Correspondência}

${ }^{1}$ Ana Rachel Fonseca de Oliveira

Universidade do Estado do Rio de Janeiro.

Rio de Janeiro, RJ.

Email: fonseca.anarachel@gmail.com

ORCID: http://orcid.org/0000-0003-3086-9156

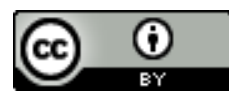

JITA: BI. User interfaces, usability.

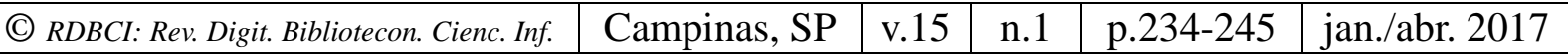




\section{INTRODUÇÂO}

A forma de acesso à Internet tem sido modificada nos últimos anos à medida que os computadores (desktops e notebooks) foram perdendo espaço por conta de suas versões móveis no formato de tablets e smartphones. Em uma apuração recente divulgada pelo Instituto Brasileiro de Geografia e Estatística (IBGE), por meio da Pesquisa Nacional Por Amostra de Domicílios (Pnad), foi constatado que $80,4 \%$ das famílias brasileiras entrevistadas utilizam o smartphone como principal meio de acesso à Internet, deixando para trás computadores, tablets, TVs inteligentes e outros equipamentos (CETIC.BR, 2016). Além de vantagens como banda larga móvel, os dispositivos móveis possuem um custo mais acessível para a maioria da população, são mais fáceis de operar, são multitarefas e são portáteis.

Atualmente, os aparelhos celulares, que antes serviam apenas para enviar e receber ligações e/ou mensagens, ganharam nova utilidade conforme suas funções foram ampliadas com serviços que possibilitam a seus usuários assistir vídeos, ler livros eletrônicos, acessar mapas, navegar nas redes sociais, compartilhar informações, e muito mais. Na rebarba da versatilidade que um aparelho móvel oferece, aliado às ferramentas da web 2.0 cujo traço principal é a colaboração e interatividade, surgiram os aplicativos (apps) desenvolvidos especialmente para estes aparelhos.

O uso de dispositivos móveis já é uma realidade no cotidiano das universidades brasileiras. Uma gama de universidades disponibilizam para professores e alunos tablets para uso pedagógico, com acesso a todo material didático dos cursos e livros eletrônicos. No entanto, muitos dos que os recebem ainda não sabem como tirar melhor proveito desses equipamentos. Atualmente, o conceito de aprendizagem móvel é discutido amplamente por pesquisadores na área da Educação objetivando modernizar, dinamizar, ampliar e enriquecer suas experiências pedagógicas.

Segundo Carvalho (1980), as bibliotecas universitárias (BUs) devem se posicionar de forma proativa procurando se integrar sempre ao ambiente universitário que as cerca. Devem avaliar seus serviços e redefini-los ao longo do tempo para que se adequem aos objetivos e atividades da própria instituição. Partindo desta afirmação, pretendemos neste trabalho aproximar a discussão sobre aprendizagem móvel das práticas biblioteconômicas. Como trabalhar todas as possibilidades de um dispositivo móvel dentro das bibliotecas universitárias?

Para tentar responder esta pergunta, apresentaremos aqui alguns aplicativos que podem ser utilizados em atividades extracurriculares. Nossa proposta, dentro da realidade de uma biblioteca universitária que atende aos usuários da área de saúde, contempla principalmente os aplicativos desta área do conhecimento, porém a ideia da utilização destas ferramentas como fontes de informação, educação e comunicação poderá ser aplicada em inúmeras outras áreas do saber.

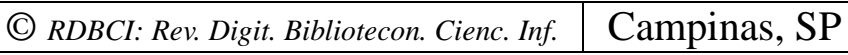

v.15 $\mid$ n.1

p.234-245

jan./abr. 2017 


\section{O BIBLIOTECÁRIO MÉDICO E SUA ATUAÇÃO NA BIBLIOTECA UNIVERSITÁRIA}

Medicina e tecnologia são áreas do conhecimento que seguem de mãos dadas ao longo dos anos, gerando avanços importantes e imprescindíveis em prol da saúde humana. Desta maneira, faz-se necessário ao bibliotecário médico manter-se competitivo e acompanhar as evoluções tecnológicas na intenção de suprir as expectativas de seus usuários. A informação para saúde tem um caráter extremamente dinâmico e mutante e o bibliotecário deverá acompanhar este ritmo em seu fazer diário.

Os desenvolvimentos tecnológicos no setor da saúde nos últimos 20 anos mudaram radicalmente a maneira de se consumir e gerenciar este tipo de informação. Posto isto, a ênfase não está mais no espaço físico da biblioteca de saúde, e sim no bibliotecário médico. (LAWTON; BURN, 2015).

Este profissional da informação atua na cooperação em diagnósticos médicos, realiza pesquisas acadêmicas em bases de dados de saúde auxiliando estudantes, docentes e pesquisadores e dissemina informações aos usuários utilizando fontes de informação de inúmeras naturezas. Nas últimas décadas, com os avanços da informação médica impulsionada pelo advento da Internet, o bibliotecário médico ganha mais uma função: a de educador.

Os estudos acerca do tema competência informacional apontam que o bibliotecário é o profissional indicado e competente para exercer a função de educador neste aspecto. Este profissional da informação deve atuar no processo de ensino-aprendizagem tornando os usuários hábeis e competentes para buscar e usar as informações. Habilidades que tornam a recuperação da informação eficiente, precisa e eficaz devem ser obrigatórias em se tratando de informação para a saúde, a qual se torna urgente e imprescindível no caso de vidas humanas.

Entendemos ainda que este tipo de educação e treinamento deve ser iniciado desde o início da graduação, formando alunos que futuramente conduzirão com propriedade uma equipe médica apta para realizar uma investigação clínica de maneira ativa e independente, já que, citando a American Library Association (1989, p.1 apud. Cunha; Orelo, 2013):

[...] para ser competente em informação a pessoa deve ser capaz de reconhecer quando precisa de informação e possuir habilidade para localizar, avaliar e usar efetivamente a informação. Para produzir esse tipo de cidadania é necessário que escolas e faculdades compreendam o conceito de competência informacional e o integrem em seus programas de ensino e que desempenhem um papel de liderança preparando indivíduos e instituições para aproveitarem as oportunidades inerentes à sociedade da informação. Em última análise, pessoas que têm competência informacional são aquelas que aprenderam a aprender. Essas pessoas sabem como aprender porque sabem como a informação está organizada, como encontrar a informação e como usar a informação, de tal forma que outros possam aprender com elas.

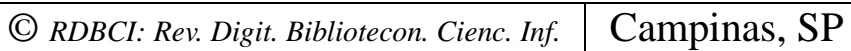

\begin{tabular}{l|l|l} 
v.15 & n. 1
\end{tabular}

p.234-245

jan./abr. 2017 
As funções de um bibliotecário médico são redesenhadas conforme as necessidades informacionais de seu público-alvo. Uma vez que o setor tecnológico tem beneficiado muito a maneira de se estudar/trabalhar no campo da saúde, observamos a tendência, principalmente através dos alunos de graduação da Universidade, de uma medicina rica em recursos eletrônicos, conectada à Internet, e repleta de funcionalidades da tecnologia da web 2.0, com uso em massa de dispositivos móveis. Sendo assim, prevemos que o próximo know-how no fazer da biblioteconomia médica será atuando na aprendizagem móvel, com conteúdos audiovisuais, e principalmente utilizando fontes de informação provenientes de aplicativos de saúde.

\title{
3 APRENDIZAGEM MóvEL
}

Segundo a definição adotada pela Organização das Nações Unidas para a Educação, Ciência e Cultura (UNESCO),

\begin{abstract}
a aprendizagem móvel envolve o uso de tecnologias móveis, isoladamente ou em combinação com outras tecnologias de informação e comunicação (TIC), a fim de permitir a aprendizagem a qualquer hora e em qualquer lugar. A aprendizagem pode ocorrer de várias formas: as pessoas podem usar aparelhos móveis para acessar recursos educacionais, conectar-se a outras pessoas ou criar conteúdos, dentro ou fora de sala de aula. A aprendizagem móvel também abrange esforços em apoio a metas educacionais amplas, como a administração eficaz de sistemas escolares e a melhor comunicação entre escolas e famílias.
\end{abstract}

As tecnologias móveis evoluem constantemente abarcando uma grande diversidade de suportes, entre eles, smartphones, tablets, leitores de livros digitais (e-readers), aparelhos portáteis de áudio e consoles manuais de videogames. De maneira geral, algumas particularidades os unem, como por exemplo, o fato de serem digitais, portáteis, de propriedade e controle de um indivíduo, todos tem acesso à Internet, são multitarefas e incluem funções de multimídia.

A aprendizagem móvel ganha um alcance quase infinito ao considerarmos a possibilidade de estender este tipo de prática para áreas físicas onde escolas, livros e computadores são insuficientes. Conforme o preço dos aparelhos móveis, principalmente smartphones, vai baixando, é possível vislumbrar um cenário de maior igualdade e oferta educacional. Outro ponto interessante desta modalidade é o imediatismo. Enquanto alunos teriam que esperar até a próxima aula para tirar dúvidas, receberem feedbacks e orientações, desta vez é possível receber uma resposta de um professor ou até mesmo de um colega de classe praticamente em tempo real.

Outro atributo favorável é a mobilidade. Os aparelhos permitem que se aprenda a qualquer hora, em qualquer lugar, fora e/ou dentro do ambiente físico de ensino. Isto permite uma autonomia maior na aprendizagem, respeitando os limites e disponibilidade do aluno, sendo possível eleger qual tipo de tarefa realizar de acordo com o tempo disponível para tal e no local e horário que desejar.

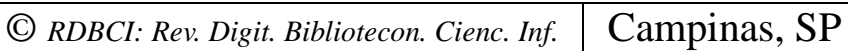

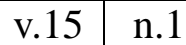

p.234-245

jan./abr. 2017 
As vantagens e benefícios da aprendizagem móvel são inumeráveis, porém é necessário que sejam adotadas diretrizes para a melhor aplicação desta variante educacional. A UNESCO (2013) elaborou um guia de Diretrizes Políticas Para Aprendizagem Móvel direcionadas para educadores, no qual destacamos os principais pontos a seguir:
a) criar ou atualizar políticas referentes à aprendizagem móvel;
b) treinar professores sobre como fazer avançar a aprendizagem por meio de tecnologias móveis;
c) fornecer apoio e formação a professores por meio de tecnologias móveis;
d) criar e aperfeiçoar conteúdos educacionais para uso em aparelhos móveis;
e) assegurar igualdade de gênero para estudantes móveis;
f) ampliar e melhorar as opções de conectividade, assegurando também a equidade;
g) desenvolver estratégias para fornecer acesso igual a todos;
h) promover o uso seguro, responsável e saudável das tecnologias móveis;
i) usar as tecnologias para melhorar a comunicação e gestão educacional.

A conjuntura atual na prática de ensino é de uma educação fortemente digital, focada no aluno e de maneira interativa e colaborativa. Os nativos digitais são atores ativos no quadro educacional, que vai desde o ensino básico até o ambiente universitário. A geração móvel de alunos, ou mais popularmente chamada "geração polegar" carece de planos curriculares que incentivem o uso de tecnologias móveis para aprendizagem e aquisição de conhecimento. Não podemos esquecer aqui que os próprios professores, docentes e demais profissionais de ensino devem passar por uma reciclagem para usufruir desta modalidade emergente em ensinança já que, na grande maioria, são "imigrantes digitais" pois não nasceram já imersos no mundo digital e ainda estão aprendendo a utilizar as novíssimas tecnologias de informação e comunicação.

Neste cenário a biblioteca deverá ser uma parceira presente e atuante fornecendo todo apoio necessário em questões informacionais e especialmente no uso destes aparelhos, como por exemplo, implantando programas de capacitação com intuito de demonstrar funcionalidades e potencialidades destas inovações.

\section{OS APLICATIVOS PARA DISPOSITIVOS MÓVEIS}

Os aplicativos (apps) integram as chamadas Novas Tecnologias de Informação e Comunicação (NTIC), e são gamas de ferramentas tecnológicas emergentes que tiraram proveito dos artifícios da web 2.0 para capturar, armazenar, recuperar, analisar, receber e compartilhar informação. Por serem tecnologias desenvolvidas para uso em aparelhos móveis, trazem consigo a possibilidade de serem personalizadas e individualizadas. O usuário poderá customizar os aplicativos baixados de acordo com suas preferências e particularidades.

Os apps são extremamente versáteis e com propósitos bastante diversificados. Muitos agregam em uma única ferramenta recursos visuais e auditivos capazes de estimular o estudo com auxilio de interfaces atraentes e extremamente amigáveis e intuitivas. Estes aplicativos podem ser baixados em plataformas diferentes, contemplando assim usuários que utilizam modelos e sistemas operacionais diversos.

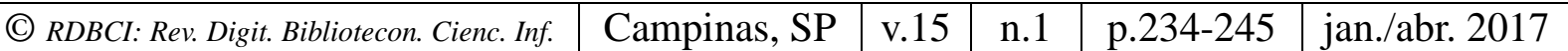


O mercado de apps tem crescido em ritmo acelerado nos últimos anos e possuem um prognóstico de se firmar na indústria de conteúdos digitais por muitos anos mais. Diariamente são criados e atualizados incontáveis aplicativos que abrangem múltiplas finalidades que vão desde entretenimento, até organização de finanças, negócios, utilização de meios de transporte, saúde, bem-estar, fitness, viagens e muito mais. É importante frisar que existem apps gratuitos e pagos. Geralmente os que demandam custos são relativamente a preços acessíveis, e seus conteúdos e qualidade não os tornam melhores que os gratuitos, apenas são mais completos em algumas especificidades.

\subsection{Os aplicativos de saúde}

Os aplicativos médicos são idealizados por uma equipe de profissionais de saúde e concretizados por profissionais de tecnologia da informação. Geralmente os apps são desenvolvidos ao longo de muitas pesquisas acadêmicas, o que daria a eles a credibilidade necessária. No entanto, a chamada área de Mobile Health, principalmente no Brasil, ainda está desprovida de mecanismos regulatórios que legitimem o uso desta tecnologia para a área de saúde.

Dentre os milhares de aplicativos de saúde hoje existentes nas principais plataformas de download, encontramos ferramentas que podem ser muito úteis nos campos de educação e informação em saúde. Existem livros e atlas de anatomia totalmente adaptados para uso em dispositivos móveis onde é possível aprender interativamente e de maneira agradável. Há ainda jogos que criam casos clínicos para que alunos e professores testem seus conhecimentos na área e mantenham-se atualizados sobre novos procedimentos médicos. Também existem em formatos de aplicativos obras de referência, bulários e prescrições médicas que podem ser consultadas a qualquer momento, em qualquer lugar.

Através de leitura em periódicos científicos, principalmente da área médica, e rankings de avaliação em sítios especializados e lojas online de aplicativos foram selecionados alguns deles que podem ser utilizados no ambiente universitário, por alunos e docentes a fim de servir como complemento pedagógico.

A proposta é que a biblioteca universitária faça um papel de mediadora entre estas fontes de informação e seus usuários em potencial. O que estamos sugerindo neste trabalho é que a biblioteca sirva como um grande observatório de monitoramento destas fontes de informação e um espaço de ensino sobre como e com qual finalidade devemos utilizar estas ferramentas. A sugestão é que ultrapassemos os limites da competência informacional focando agora em uma app literacy

De acordo com Cerigatto (2016), "na sociedade do século XXI, em especial aos ambientes educativos, todos os contextos estão sujeitos à aprendizagem. Destes contextos, surgem novas literacias [...]”. O número de usuários de aplicativos como fontes de informação aumenta conforme estas ferramentas são criadas de maneira que consigam suprir boa parte

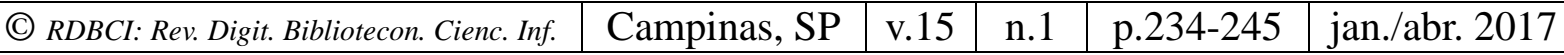


das informações desejadas. Uma pesquisa realizada na Inglaterra sobre os hábitos de consumo de informação entre residentes e alunos de Medicina atesta que boa parte deles considera os aplicativos como uma ferramenta valiosa para suporte clínico e recursos educacionais. (CHAMBERLAIN et al, 2015). Com base nestas afirmações, entendemos ser necessário capacitar os usuários de apps de saúde para reconhecimento e melhor aproveitamento destas fontes informacionais.

A cada dia surgem novos aplicativos no mercado, os números são gigantescos, um verdadeiro "app overload" (VAN VELSEN et al, 2013) que acaba por inundar e confundir seus usuários. Dentro dos milhares existentes, os aplicativos de saúde ocupam uma grande fatia deste mercado. Por isso torna-se fundamental acompanhar estas evoluções de forma que seja possível ainda identificar e avaliar os melhores e mais completos mecanismos tecnológicos neste aspecto. Para mais, examinar a qualidade da informação que permeiam estas ferramentas é imprescindível para que sejam suficientemente confiáveis para serem incluídos como fontes de informação do plano de estudos propostos pela universidade.

\subsection{Aplicativos para educação e informação em saúde}

No quadro a seguir listamos aplicativos que podem e devem ser explorados como complementos extraclasse. Foram selecionados 25 fontes de informação para exemplificar o vasto alcance e possibilidades que estas ferramentas podem atingir nos campos da educação em saúde.

Quadro 1. Lista de apps de saúde com funcionalidades educativas

\begin{tabular}{|l|l|}
\hline \multicolumn{1}{|c|}{ Título } & \multicolumn{1}{c|}{ Breve descrição } \\
\hline BMJ OnExamination & $\begin{array}{l}\text { Quiz de perguntas e respostas de } \\
\text { conhecimentos médicos }\end{array}$ \\
\hline Bulário Digital & Banco de dados de bulas de remédio \\
\hline Bulário veterinário & Banco de dados de bulas veterinárias \\
\hline Calculate by QxMD & Calculadora médica \\
\hline CID 10 & $\begin{array}{l}\text { Consulta rápida e inteligente aos códigos e } \\
\text { descrições completas da CID10 }\end{array}$ \\
\hline Clinical Key & Pesquisa clínica baseada em evidências \\
\hline Clinical Sense & $\begin{array}{l}\text { Jogo interativo para tomada de decisões } \\
\text { clínicas }\end{array}$ \\
\hline DailyRounds - Doctor's App & Compartilhamento e testes de casos clínicos \\
\hline Epocrates Plus & Referência em informação clínica \\
\hline Figure 1 - Imagens médicas & $\begin{array}{l}\text { Compartilhamento de casos clínicos através } \\
\text { de fotografias }\end{array}$ \\
\hline Human Anatomy Atlas & Atlas de anatomia humana em 3D \\
\hline JoVE - Journal of Visualized Experiments & Periódico em vídeo revisado por pares \\
\hline Leia por QxMD & Revista digital de literatura médica \\
\hline Medical News Online & Notícias médicas \\
\hline Medscape & Referência em informação clínica \\
\hline
\end{tabular}

${ }^{1}$ Sobrecarga de aplicativos.

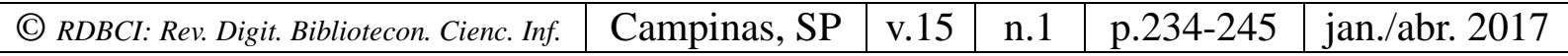




\begin{tabular}{|l|l|}
\hline MedQuiz Residência Médica & Questões de prova de Residência Médica \\
\hline Prognosis: Your Diagnosis & Jogo interativo de simulação de casos clínicos \\
\hline PubMed Mobile & Referência em publicaços científicas \\
\hline Sobotta Anatomy Atlas & Atlas de anatomia humana \\
\hline Terminologia médica & Dicionário médico \\
\hline The Lancet & Periódico científico \\
\hline Touch Surgery & Simulador de cirurgia \\
\hline UpToDate & Decisões médicas baseadas em evidência \\
\hline WebMD & $\begin{array}{l}\text { Informação em saúde e apoio à tomada de } \\
\text { decisão clínica }\end{array}$ \\
\hline WhiteBook: clinical decision & $\begin{array}{l}\text { Guia de prescrições médicas e condutas } \\
\text { clínicas e tomadas de decisão }\end{array}$ \\
\hline
\end{tabular}

Fonte: A autora, 2016.

A título de ilustração capturamos as telas de alguns aplicativos, apresentados nas figuras 1, 2 e 3, para exemplificar a gama de possibilidades que podem ser disponibilizadas. .
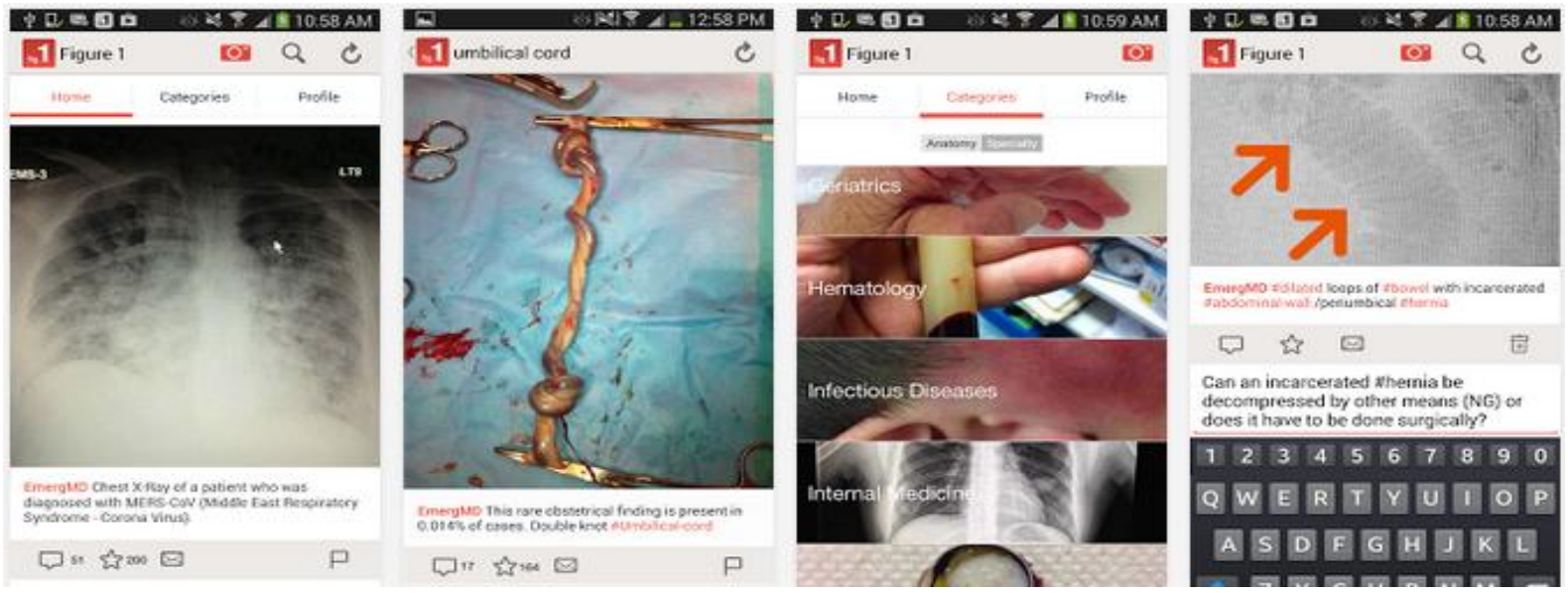

Figura 1. Telas do Figure 1

Fonte: Google Play Store, 2016.

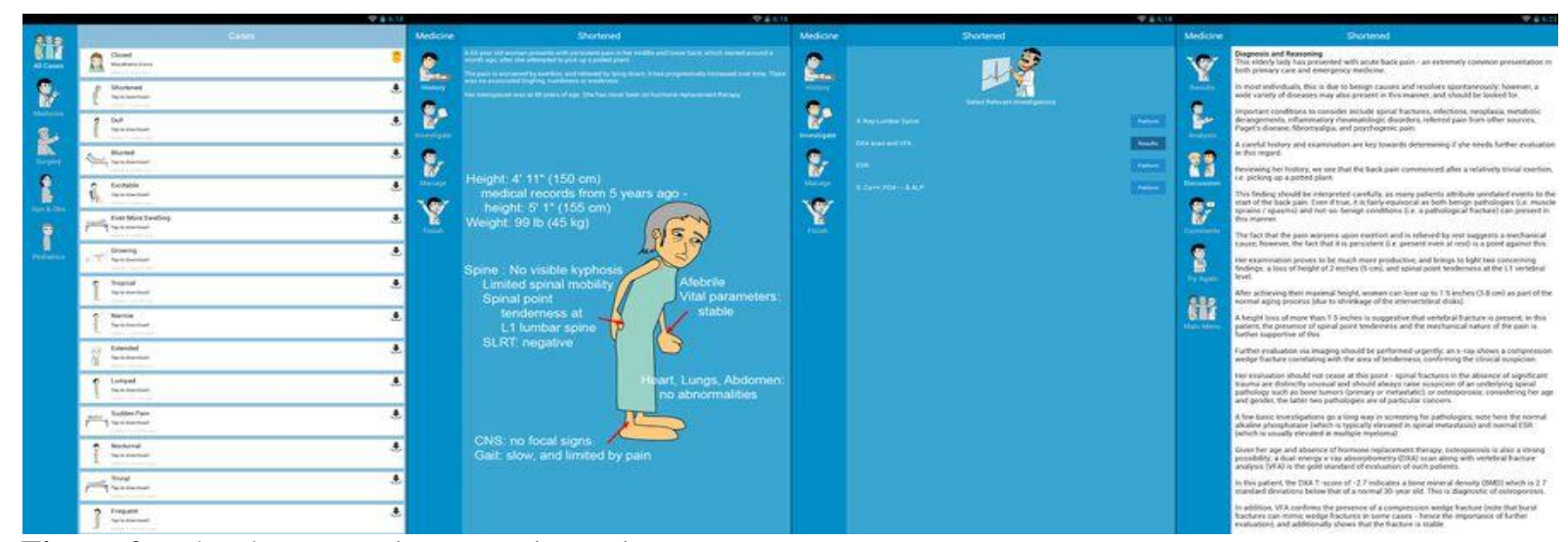

Figura 2. Telas do Prognosis: Your Diagnosis

Fonte: Google Play Store, 2016.

\begin{tabular}{l|l|l|l|l|l}
\hline (C) RDBCI: Rev. Digit. Bibliotecon. Cienc. Inf. & Campinas, SP & v.15 & n.1 & p.234-245 & jan./abr. 2017 \\
\hline
\end{tabular} 


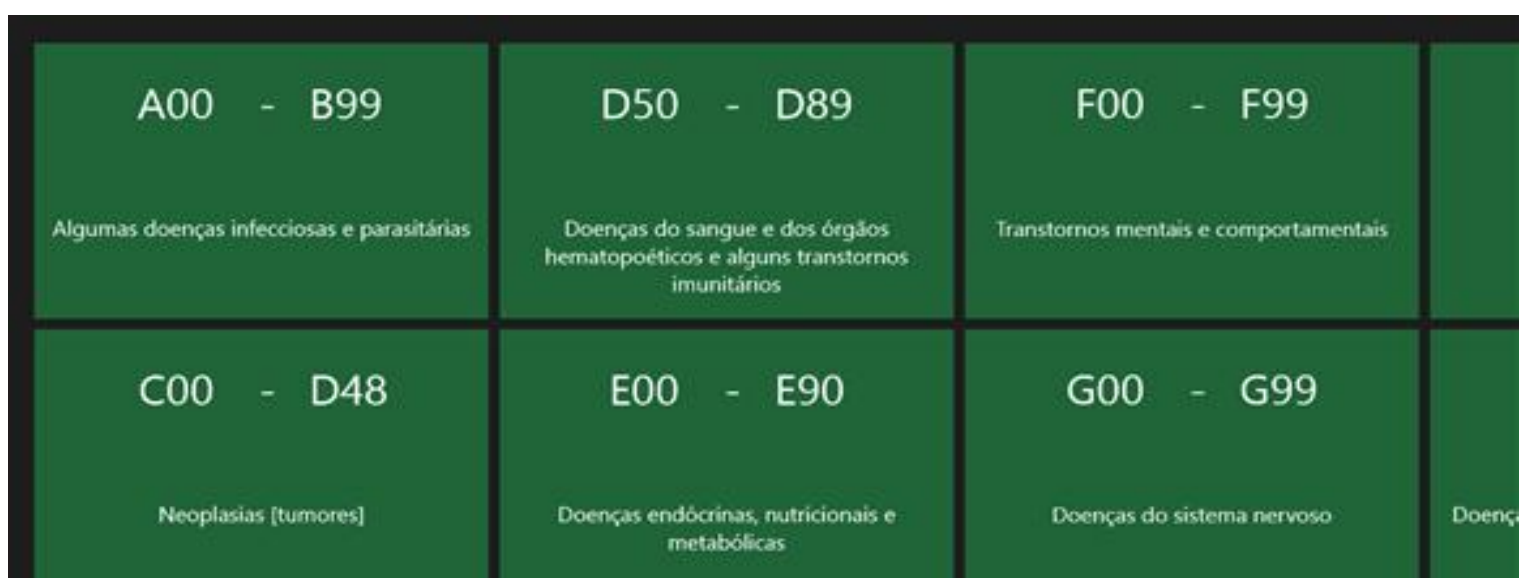

Figura 3. Tela do CID 10

Fonte: Google Play Store, 2016.

\section{CONSIDERAÇÕES FINAIS}

As novas tecnologias de informação e comunicação utilizadas por meio de dispositivos móveis são atualmente o grande trunfo para uma renovação do contexto educacional com objetivo de favorecer uma geração de alunos que nasceu literalmente conectada à tecnologia. Com o surgimento de novas fontes de informação que emergiram paralelamente à tecnologia da web 2.0, como é o caso dos aplicativos, é mister que façamos contribuir com novos letramentos, ou seja, formar indivíduos capazes de "aprender a aprender" e fazer uso efetivo das recentes origens informacionais que permeiam o atual ecossistema da informação. A biblioteca universitária deve servir de ponte entre estas ferramentas e seus usuários em potencial, lançando mão de seu espaço físico e de seus profissionais qualificados para criar um ambiente de descobertas, monitoramento, treinamento, e por que não de criação de conteúdos digitais próprios?

O bibliotecário, sendo ator efetivo na prática pedagógica, deve aproveitar ao máximo as vantagens oferecidas pelas tecnologias móveis e seus instrumentos, capacitando docentes e discentes de modo que estes possam incorporar e usufruir com sucesso as emergências tecnológicas em benefício do ensino/aprendizagem. Esse campo ainda carece de muitas pesquisas, trocas de experiências e práticas inovadoras.

Quando apresentamos aos alunos e professores as contemporaneidades tecnológicas educacionais, ambos ganham novas competências que futuramente serão aplicadas com sucesso em seus ambientes de trabalho. Ademais, os aplicativos que podem ser utilizados na prática educativa aumentam a eficiência de educadores e alunos por automatizar a distribuição, a coleta, a verificação e a documentação de avaliações e exames. 


\section{EL USO DE APLICACIONES DE SALUD PARA DISPOSITIVOS MÓVILES COMO FUENTES DE INFORMACIÓN Y EDUCACIÓN SANITÁRIA}

\section{RESUMEN}

Aplicaciones para dispositivos móviles son nuevas fuentes de información fuertemente usadas por individuos hiperconectados, llamados "generación pulgar", que además de su uso recreativo se haga uso de estas tecnologías móviles en un contexto educativo innovando el esquema de enseñanza-aprendizaje. El artículo propone que la biblioteca universitaria se utiliza como espacio de enseñanza y fomenta el uso de los dispositivos móviles como herramientas educativas ya que el uso masivo de las tabletas y especialmente de los teléfonos inteligentes, refuerzan el concepto de aprendizaje móvil y amplian sus posibles funciones dentro del espacio de la biblioteca física. De hecho, una biblioteca universitaria que se centra en los estudiantes de la área de la salud y, principalmente, de la Medicina, se centrará en aplicaciones de salud como fuentes de información, comunicación y educación. Dentro de este escenario de innovaciones tecnológicas que modifican las formas de acceso y uso de la información científica, el bibliotecario debe asumir el papel de mediador y facilitador entre estos avances tecnológicos y sus potenciales usuarios.

PALABRAS CLAVE: Aplicaciones móviles. Aprendizaje móvil. Alfabetización informacional. Dispositivos móviles.

Submetido em: $15 / 01 / 2017$

Aceito em: 20/01/2017

Publicado em: 31/01/2017

\section{REFERÊNCIAS}

AGGARWAL, Mayank; MATHUR, Akshay; KAREL, Yatin. Doctor App: A life saving Mobile Application. International Journal Of Computer Science and Information Technologies, v.5, n. 5, p.6290-6295, 2014. Disponível em: $<$ http://citeseerx.ist.psu.edu/viewdoc/download?doi=10.1.1.660.5973\&rep=rep1\&type=pdf $>$ Acesso em: 20 ago. 2015.

AZEVEDO, Alexander Willian; BERAQUET, Vera Silvia Marão Fabiana Araujo. Formação e competência informacional do bibliotecário médico brasileiro. RDBCI: Revista Digital de Biblioteconomia e Ciência da Informação, v. 7, n. 2, p. 199-218, 2010. Disponível em: $\langle$ http://periodicos.sbu.unicamp.br/ojs/index.php/rdbci/article/view/1964>. Acesso em: 17 mar. 2016.

BECKER, Stefan et al. mHealth 2.0: experiences, possibilities, and perspectives. JMIR mHealth and uHealth, v. 2, n. 2, p. e24, 2014. Disponível em: <http://mhealth.jmir.org/2014/2/e24/?trendmd-shared=0> Acesso em: 3 dez. 2015.

BORUFF, Jill ; STORIE, Dale. Mobile devices in medicine: a survey of how medical students, residents, and faculty use smartphones and other mobile devices to find information. Journal of the Medical Library Association, v. 102, n. 1, p. 22-39, 2014. Disponível em:

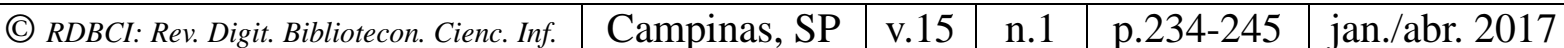


$<$ http://go.galegroup.com/ps/anonymous?id=GALE\%7CA361185587\&sid=googleScholar\&v $=2.1 \& \mathrm{it}=\mathrm{r} \&$ linkaccess $=$ fulltext \&issn $=15365050 \& \mathrm{p}=\mathrm{AONE} \& \mathrm{sw}=\mathrm{w} \&$ authCount=1\&isAnony mousEntry=true $>$ Acesso em: 31 mar. 2015.

CARVALHO, Maria Carmen Romcy de. Estabelecimento de padrões para bibliotecas universitárias. Brasília: ABDF, 1981.

CAVALCANTE, Lídia Eugenia et al. Competência em informação na área da Saúde. InCID: Revista de Ciência da Informação e Documentação, v. 3, n. 1, p. 87-104, 2012. Disponível em: 〈http://www.revistas.usp.br/incid/article/viewFile/42372/46043>. Acesso em: 20 ago. 2016.

CENTRO REGIONAL DE ESTUDOS PARA O DESENVOLVIMENTO DA SOCIEDADE DA INFORMAÇÃO (CETIC.BR.) TIC Governo Eletrônico 2015: órgãos públicos federais e estaduais. Disponível em: $\langle$ http://cetic.br/pesquisa/governo-eletronico/indicadores $\rangle$. Acesso em: 2 maio 2016.

CERIGATTO, Mariana Pícaro. Novos letramentos da informação. Anais do Encontro Internacional Tecnologia, Comunicação e Ciência Cognitiva, v. 2, n. 1, 2016. Disponível em: http://www.anais.tecccog.net/index.php/anais/article/view/52. Acesso em: 5 maio 2016.

CHAMBERLAIN, David; ELCOCK, Martin; PULIGARI, Preeti. The use of mobile technology in health libraries: a summary of a UK-based survey. Health Information \& Libraries Journal, v. 32, n. 4, p. 265-275, 2015.

CUNHA, Miriam Figueiredo Vieira da; ORELO, Eliane Rodrigues Mota. O bibliotecário e a competência informacional. Informação \& Sociedade, v. 23, n.2, p.25-32, maio/ago. 2013. Disponível em: <http://www.ies.ufpb.br/ojs/index.php/ies/article/view/12892 >. Acesso em: 23 nov. 2015.

DAVIES, Bethany S. et al. Mobile Medical Education (MoMEd)-how mobile information resources contribute to learning for undergraduate clinical students-a mixed methods study. BMC medical education, v. 12, n. 1, p. 1, 2012. Disponível em: $\langle$ http://citeseerx.ist.psu.edu/viewdoc/download?doi=10.1.1.270.7450\&rep=rep1\&type=pdf $>$ Acesso em: 7 out. 2015.

GOOGLE Play Store. Loja de aplicativos. Disponível em: 〈https://play.google.com/store〉 Acesso em: 5 maio 2016.

LAWTON, Aoife; BURNS, Jane. A review of competencies needed for health librarians: a comparison of Irish and international practice. Health Information \& Libraries Journal, v. 32, n. 2, p. 84-94, 2015. Disponível em:

<http://onlinelibrary.wiley.com/doi/10.1111/hir.12093/abstract;jsessionid=6FF8876D2A8646 C548C80B4B120D5429.f04t04> Acesso em: 14 abr. 2016.

MOURA, Adelina. Geração móvel: um ambiente de aprendizagem suportado por tecnologias móveis para a "Geração Polegar". In: Actas da VI Conferência Internacional de TIC na Educação - Desafios 2009. Braga: Universidade do Minho, 2009. p. 50-78. Disponível em: $<$ http://repositorio.uportu.pt/jspui/bitstream/11328/472/1/Gera\%C3\%A7\%C3\%A30\%20M\%C 3\%B3vel\%282009\%29.pdf>. Acesso em: 18 jan. 2016.

\begin{tabular}{l|l|l|l|l|l} 
(C) RDBCI: Rev. Digit. Bibliotecon. Cienc. Inf. & Campinas, SP & v.15 & n.1 & p.234-245 & jan./abr. 2017 \\
\hline
\end{tabular} 
UNESCO. Diretrizes Políticas Para Aprendizagem Móvel. France: UNESCO, 2013.

Disponível em: 〈http://unesdoc.unesco.org/images/0022/002277/227770por.pdf〉 Acesso em: 14 jun. 2015.

VAN VELSEN, Lex; BEAUJEAN, Desirée JMA; VAN GEMERT-PIJNEN, Julia EWC. Why mobile health app overload drives us crazy, and how to restore the sanity. BMC medical informatics and decision making, v. 13, n 1, p. 1, 2013.

WALLACE, Sean; CLARK, Marcia; WHITE, Jonathan. 'It's on my iPhone': attitudes to the use of mobile computing devices in medical education, a mixed-methods study. BMJ open, v. 2, n. 4, p. e001099, 2012. Disponível em:

$\langle$ http://citeseerx.ist.psu.edu/viewdoc/download?doi=10.1.1.690.5076\&rep=rep1\&type=pdf $>$ Acesso em: 5 set. 2015.

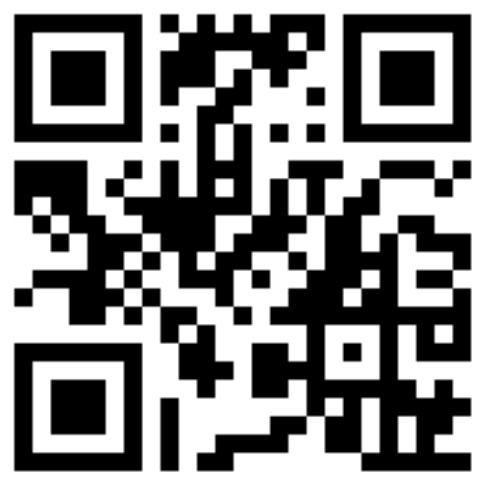

